\title{
TROPOLITE, on the path of Atmospheric Chemistry Made Simple
}

\author{
Luca Maresi ${ }^{\mathrm{a}}$, Wencke Van Der Meulen ${ }^{\mathrm{b}}$, Rob Vink $^{\mathrm{c}}$ \\ ${ }^{a}$ European Space Agency, Keplerlaan 1, 2200 AG Noordwijk, - The Netherlands, \\ ${ }^{\mathrm{b}}$ Netherlands Space Office, Prinses Beatrixlaan 2, 2595 AL Den Haag - The Netherlands, \\ ${ }^{\mathrm{c}}$ TNO Stieltjesweg 1, 2628CK, Delft - The Netherlands
}

\begin{abstract}
Accurate, reliable and stable long term measurements of Earth's Atmospheric Chemistry from Space are currently done by complex instruments, whose mass is in excess of $100 \mathrm{Kg}$. TROPOMI is the more recent instrument being developed jointly by ESA and NSO and due for launch in 2015. TROPOMI, consisting of four spectrometers ranging from UV to SWIR, is paving the way to the development of high performance spectrometers that will compose the backbone of the European Copernicus system. The objective of TROPOMI is to measure trace gases with an accuracy one order of magnitude better of what is currently done from Space. While teams of engineers are still busy finalizing TROPOMI, ESA, NSO, and TNO have launched an initiative along a different development axis: to explore the possibility of a lighter version of TROPOMI, to address a market valuing a cost effective instrument for Atmospheric Chemistry.

TROPOLITE, as it is dubbed, leverages on all the technology developments and the lessons learnt from TROPOMI, but with the clear objective of a design to cost solution. Furthermore, mass and power of the instrument shall be within the envelope of a payload of a small satellite, namely $20 \mathrm{~kg}$ and $30 \mathrm{~W}$ and possibly within a volume of $20 \mathrm{x} 20 \mathrm{x} 40 \mathrm{~cm} 3$. The scope of TROPOLITE is to address a larger user base that is interested in an affordable instrument to perform from a small satellite some specific tasks relevant to Air Quality and/or Climate.

The paper, after a short overview of the TROPOMI design and current status, presents the design philosophy of TROPOLITE, and shows what are the technologies and processes stemming from the experience gained with TROPOMI that make possible a simplified, but still very performing, version of TROPOMI. A comparison in terms of performance and functionalities of the two instruments is discussed. Finally, the development plan from the current development status of TROPOLITE up to Qualification Model is presented.
\end{abstract}

Keywords: Grating Spectrometers, Remote Sensing, Atmospheric Chemistry.

\section{INTRODUCTION}

Observations from space of gases of Earth's atmosphere have over 30 years of history. These observations have been motivated by scientific research and by concerns of the effects of human activities on the environment. They have greatly improved our understanding of processes governing stratospheric ozone depletion, climate change and transportation of pollutants. Long-term continuous measurements of atmospheric trace gas have been limited to stratospheric ozone and to a few related species. Reliable long-term space-based monitoring of atmospheric constituents with accuracy sufficient to serve atmospheric chemistry applications still needs to be established. In spite of the long history on stratospheric measurements, retrieval of accurate information of the trace gases in the troposphere is still in its infancy. Only a handful of instruments have been launched to perform these measurements, namely OMI, TOMS, GOME, and Sciamachy.

One more instrument dubbed TROPOMI, (Troposphere Monitoring Instrument) is being jointly developed by ESA and The Netherlands Space Office (NSO). The instrument is scheduled for launch on 2016 on board Sentinel 5P. The objective of the mission is to improve the current capabilities to measure concentration of trace gases, among which $\mathrm{NO}_{2}, \mathrm{SO}_{2}, \mathrm{BrO}$, methane, formaldehyde. The measurements of TROPOMI, with its improved horizontal and vertical resolution, will be used in several fields as enforcement of international agreements on air quality, to study chemical

Sensors, Systems, and Next-Generation Satellites XVIII, edited by Roland Meynart, Steven P. Neeck, Haruhisa Shimoda, Proc. of SPIE Vol. 9241, 92410J · c 2014 SPIE CCC code: $0277-786 \mathrm{X} / 14 / \$ 18 \cdot$ doi: $10.1117 / 12.2067473$ 
processes and heat exchanges in the lower atmosphere, and consequently improving the accuracy of numerical weather forecasts and climate models.

The experience built on the TROPOMI project, in testing phase at the moment of writing, has already brought technology developments and a better understanding of the performance attainable with this type of configuration. The know-how built on TROPOMI and the recent technology developments provide enough confidence that performance close to TROPOMI can be achieved with an instrument with smaller mass and volume.

In the opinion of the Authors, a more affordable instrument that could fit within the constraints of a small satellite could have a larger user base, seen the multiple applications that this type of instrument can serve. A project nicknamed TROPOLITE, to outline the objective of achieving a 'lighter' version of TROPOMI, has been launched with the objective of verifying to what extend an instrument using the technologies developed within the TROPOMI project could be rescaled to fit within the volume allocated to a payload on by a small platform. The study work uses a design-to-cost approach to conceive an instrument with low recurring cost. Furthermore, the technology solutions are selected to provide the modularity for an easy customizable instrument that can fulfill the requirements of different users.

\section{DESIGN APPROACH}

The design guidelines to define the configuration of TROPOLITE has been

a) To achieve the SNR and spectral resolution of TROPOMI using a fraction of the engineering budgets.

b) To identify cost saving options, even at the expenses of reduced functionalities.

c) To obtain a modular and scalable configuration to serve different users.

d) To minimize the recurrent cost.

Point a): The design goal is to keep the engineering budgets of the instrument within $20 \mathrm{Kg}$ mass, an envelope less than a volume of $20 \times 20 \times 40 \mathrm{~cm} 3$, and using less that $30 \mathrm{~W}$. These engineering budgets stem from typical small satellite platforms whose pointing capabilities and data link are enough to operate an instrument for Earth remote sensing.

Point b): It gives the design team the freedom to greatly simplify the instrument, without compromise the quality of the products generated by the instrument. Lesser functionalities could be compensated by a smarter use of the instrument, in case of a single stream mission using an agile small platform. One example is the calibration unit of TROPOMI, quite bulky and costly, that could be simplified by using the pointing capability of the platform.

Point c): TROPOMI uses four spectrometers to cover the required spectral range. The objective of TROPOLITE is to achieve a design where more spectrometers can be stacked together with no mechanical changes to the housing. In this way a user interested only in a sub-set of the products generated by TROPOMI can select which spectrometer to embark.

Point d): The design of optical elements, the selection of the EEE components, of the cooling systems and of the assembly procedure is conceived to minimize the recurrent cost.

\section{TROPOMI AS STARTING POINT}

$\mathrm{TROPOMI}^{[1]}$ is a pushbroom imaging spectrometer with four spectrometers covering the spectral range from UV to short wave infrared (SWIR). The ground sampling distance and the spectral range are greatly enhanced with respect to its predecessors, as shown Figure 1 and Figure 2.

Figure 3 shows a functional diagram of the instrument. The instrument has a telescope and a calibration system common to all spectrometers. The spectrometers covering the UV, the visible and the NIR spectral range are assembled in a single mechanical part, nicknamed 'the motor block' as its many holes and cavities resembles a motor of a car engine (Figure 4). This solution, that entails a very laborious manufacturing, has the advantage to greatly simplify the alignment of the many optical components and provides high thermal and mechanical stability to ensure the alignment of the spectral 
channels. The telescope is equipped with a polarization scrambler to minimize the sensitivity to the polarization state of the incoming light. The light from the telescope is separated in the flight direction by a slit which has a reflecting edge.

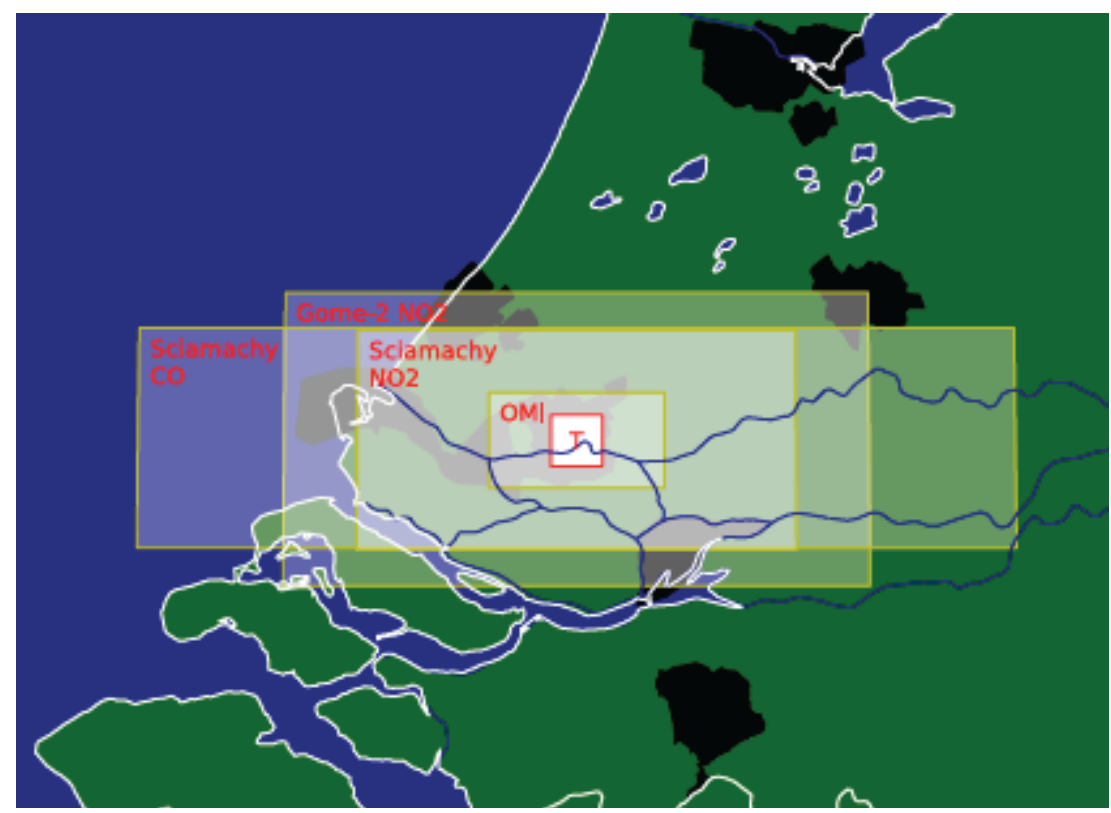

Figure 1 - Comparison of Ground Sampling Distances of Sciamachy, Gome, OMI, and TROPOMI (white square)

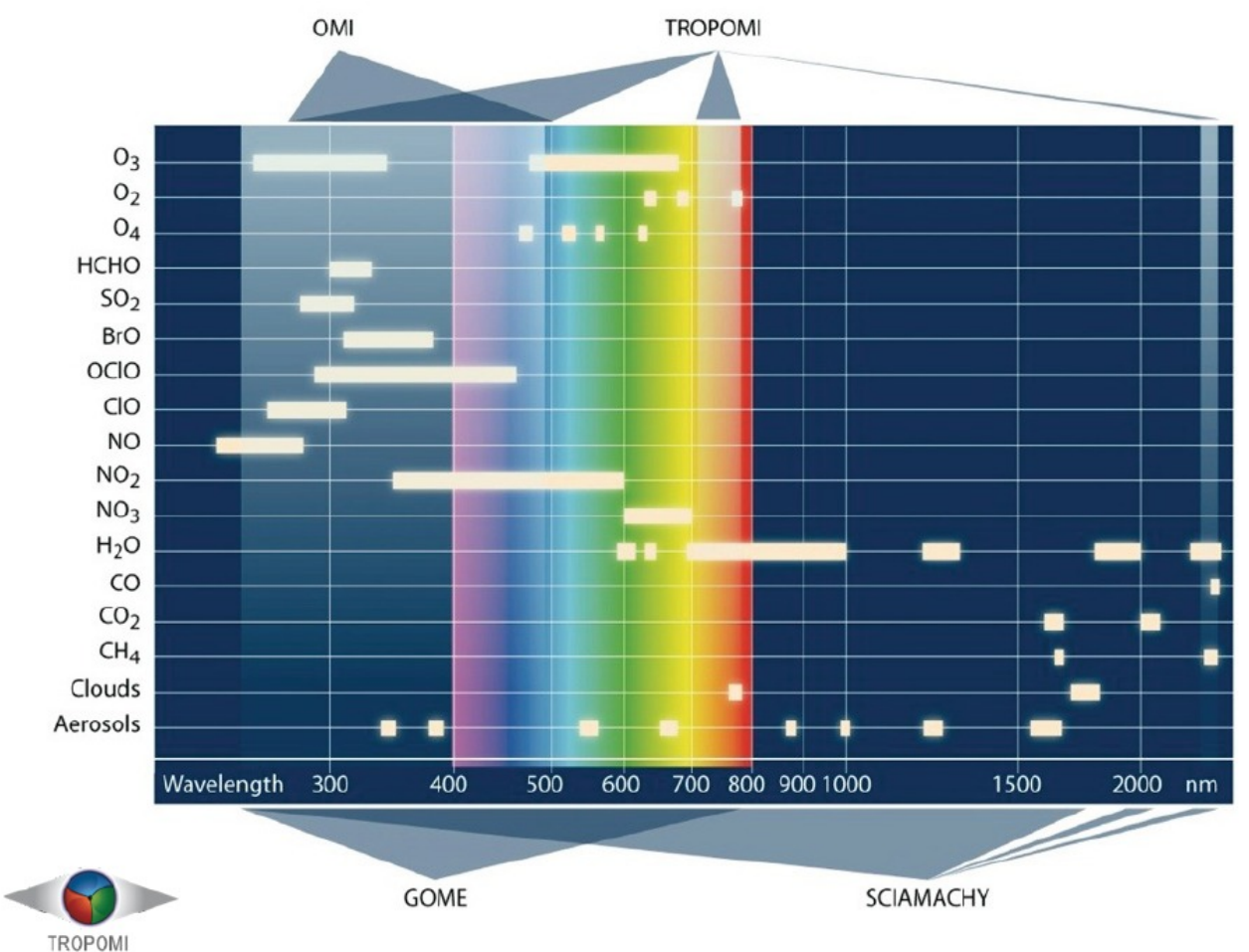

Figure 2 - Spectral Coverage of Sciamachy, Gome, OMI, and TROPOMI 
The consequence is that the UV and SWIR bands will see a slightly shifted part of the Earth as compared to the visible and NIR channels (Figure 6).

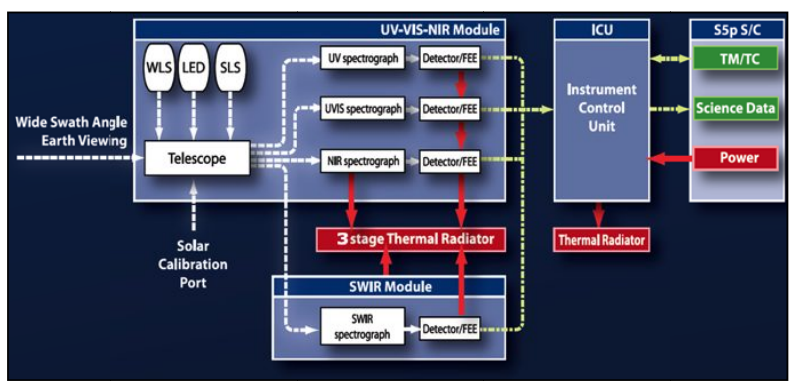

Figure 3 - TROPOMI functional diagram

The projection of the spectrometer slit on-ground has a curved shape, called spatial smile, caused by use of an off-axis telescope. The NIR and visible spectrometers use a common slit, while the SWIR and UV spectrometers are in-field separated of approximately 1 degree along the flight direction. The detectors are cooled to $\sim 210 \mathrm{~K}$ to reduce the dark current contribution and they will be thermally isolated from the remainder of the detector modules, which are kept at room temperature.

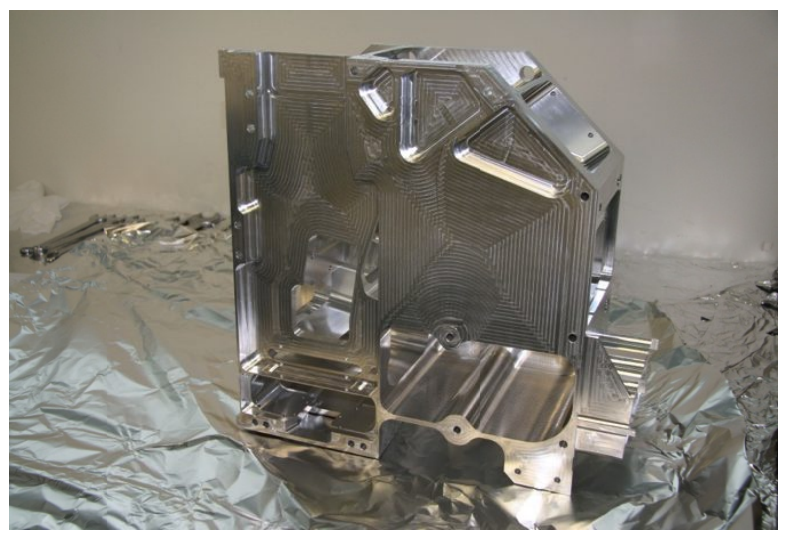

Figure 4 - UVN Mechanical Housing

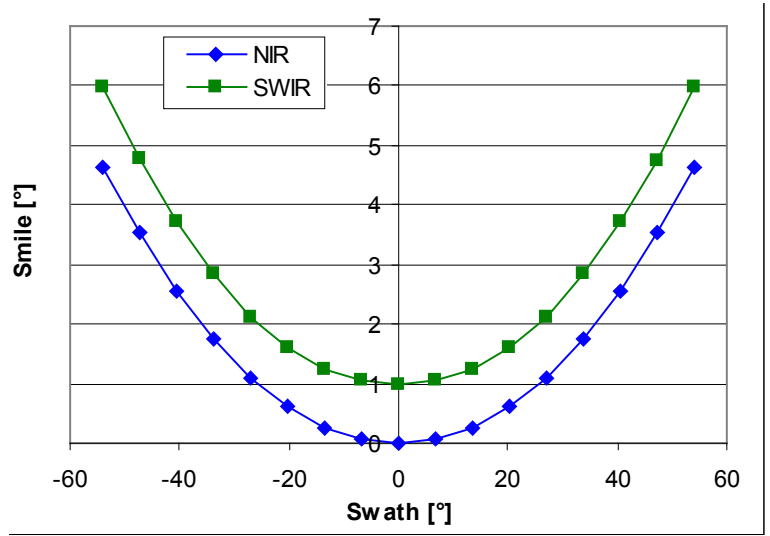

Figure 5 - On Ground Slits Projection 
The SWIR spectrometer shares the telescope the UV, visible and NIR spectrometers and receives the light via an optical relay. The SWIR optics is cooled at $200 \mathrm{~K}$ to reduce thermal self-emission and the detector is cooled at $140 \mathrm{~K}$ to suppress dark current. The SWIR spectrometer uses an immersed grating ( Figure 6) to reduce the SWIR overall volume.

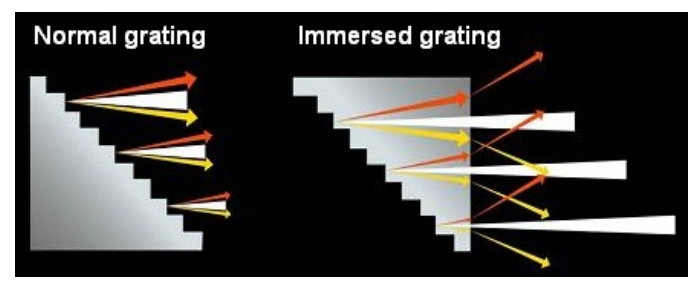

Figure 6 - Standard and Immerse Grating

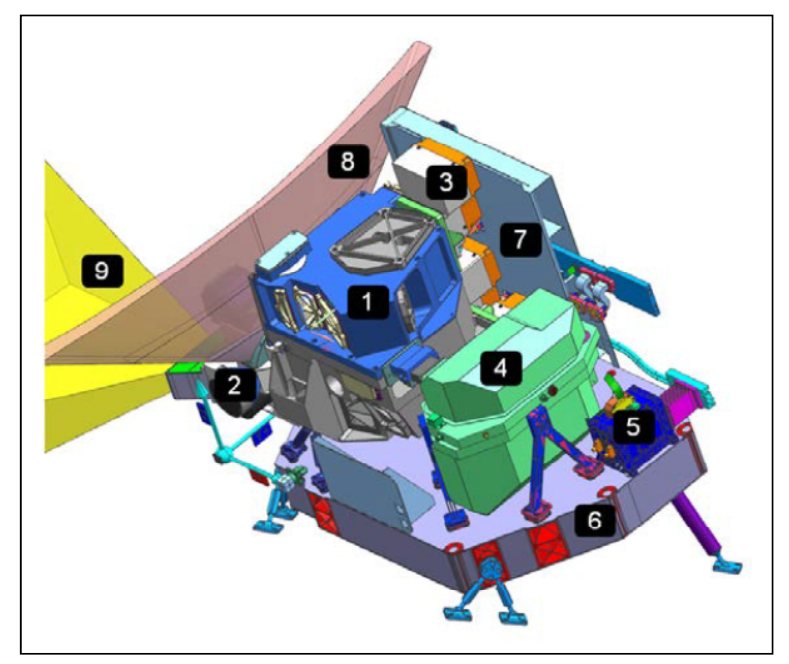

Figure 7 - TROPOMI Mounted on the TSS

The instrument control unit (ICU) provides power to the subsystems, receives tele-commands, send telemetries, and handle the processes to generate science data packets.

TROPOMI is mounted on a telescope support structure, which in turn is mounted on the spacecraft. A passive thermal radiator is used to remove heat from the system. Figure 7 shows TROPOMI located on the telescope support structure: (1) UV, UVIS and NIR Module, (2) Control Unit, (3) UVN Digital Electronics Module, (4) SWIR Module, (5) SWIR Front End Electronics, (6) Telescope Support Structure, (7) Thermal Bus Unit, (8) Nadir Field of View, (9) Field of View of the Sun Calibration Port. The calibration unit includes the following elements:

- Two Sun diffusers: one for regular use, one for degradation monitoring.

- A white light source (WLS) used for measurements of photo response non-uniformity (PRNU), calibration, and on-ground health checks.

- A LED to monitor the short term variation in the output of the WLS.

- Five laser diodes are placed in the calibration unit of the SWIR band to monitor the instrument spectral response function.

Each detector can be directly illuminated by two LEDs to check the detector response and its linearity. 


\section{TROPOMI TECHNOLOGY PLATFORM}

An important step for a design evolution is to clearly identify what is the technology platform on which to build the next generation. Clearly understand on what the new design may leverage minimize the development risks and gives the larger innovation step. The TROPOLITE instrument will surely leverage on the technology development performed for manufacturing of the free form mirrors used in the TROPOMI telescope and on the experience gained in the metrology and AIV of non-rotationally symmetric mirrors. The spectro-radiometric calibration of the instrument is one more area where TROPOMI and its predecessors have provided a solid experience. Given that the calibration of the full instrument is on the critical path, and in the case of the TROPOMI project it became one of the cost drivers, a design to cost entails a rethink of the calibration approach to keep it to a bare minimum but without jeopardizing the capability of producing quality data, that greatly depends on the knowledge of the Instrument Spectral Response Function and of other instrument parameters, that can only be measured during the on-ground calibration campaign.

The capability to manufacture free form mirrors with low roughness is probably one of the most important achievements stemming from the TROPOMI development. This technology was only used for the telescope of TROPOMI where a larger mirror micro-roughness was better tolerated. The stringent straylight requirements of these type of instruments imposes a roughness less than $2 \mathrm{~nm}$ for the optical elements of the spectrometer. This is routinely achieved on high quality spherical lenses, and, at the expenses of manufacturing cost, also on aspherical quartz lenses, but it was beyond reach for free form mirrors when the TROPOMI project started. The development done within the TROPOMI project on the improvement of the manufacturing/metrology loop achieved with the NANOMEFOS ${ }^{[2]}$ and a better predictability of the whole manufacturing process makes now possible a spectrometer configuration using only reflective elements.

\section{TROPOLITE CONFIGURATION}

\subsection{A fully reflective design}

An all reflective design as been explored by leveraging as much as possible of the recently improved capabilities to design and manufacturing free form mirrors ${ }^{[3]}$. A fully reflective spectrometer eliminates the wavelength dependence of the optical design and by using free form mirrors it is possible to significantly reduce the number of optical elements. In the current configuration TROPOLITE (Figure 8) spectrometers uses only 3 mirrors with power. All spectrometers operating from the UV to the NIR range could use the same optical design. The different wavelength ranges are implemented only by changing the grating and some of the coatings.

This solution greatly reduces the complexity and the manufacturing cost. It is scalable and can be easily customizable to match the requirements of different users: the spectral range and the spectral resolution of each spectrometer can be fine tuned only by selecting the appropriate grating. Furthermore, a number of similar spectrometers can be piled together in a fashion such that they use a shared telescope, or each spectrometer can be built as a self-standing unit with its own telescope and polarization scrambler. The selection of having one or multiple telescopes is based on the number of channels to be implemented. A configuration for a user interested in only two spectrometers, e.g. UV and NIR, will use a solution with one telescope shared by two spectrometers. In case a user is interested in a larger number of spectrometers, e.g. five spectrometers to cover to full range from UV to NIR, two or more telescope may be used to reduce the complexity of the coating, of the mechanical mounting. Furthermore, the manufacturing, test and calibration of two or three self-standing units will be faster and simpler, as the different units could be built and tested in parallel.

\subsection{In-orbit Radiometric Calibration}

Space instruments are designed to be thermally and mechanically stable and have minimal in orbit variations, but the level of accuracy required to the instruments dedicated to measurement of atmospheric gases makes them sensitive to any variation that may occur during the launch and throughout the lifetime of the instrument. Furthermore, these instruments need to perform periodical measurement of the Sun irradiance to offset the natural variation of the Sun irradiance. Therefore, most of space instruments are equipped with on-board monitoring and calibration hardware to measure different parameters, as the spectral and radiometric stability, and the variation of detector dark current. 
In instruments like OMI, Sciamachy and TROPOMI, the on-board calibration hardware is implemented as a full aperture and covers the full field of view. This solution results in a complex and bulky system with one of more moving parts used to position the calibration unit in front of the telescope. The calibration unit is a non-negligible subsystem of the instrument, and is a single point failure; therefore its reliability requirements make it a costly item.

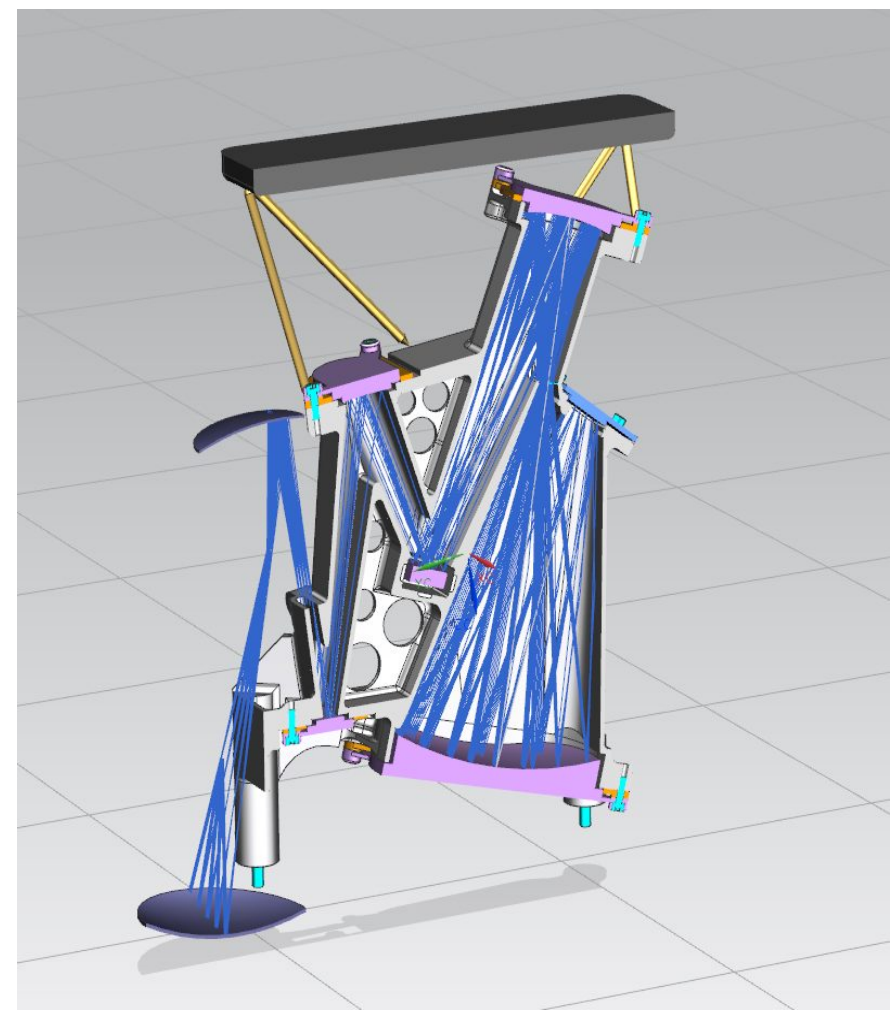

Figure 8 - TROPOLITE Optical Configuration

Following the design guideline to keep the instrument very simple, a solution with no moving part has been studied, with the objective to reach the same on-board calibration capability not to hamper the quality of the final data.

Vicarious calibrations use areas on Earth with well-known reflectance and are currently used by some mission, as Vegetation and more recently Proba-V. These areas are usually salt lakes, but the radiometric accuracy achieved with vicarious calibration is approximately $3 \%$, and it does not seems feasible to improve the performance of this type of calibration method to match the requirements of less that $1 \%$, typical of an instrument for atmospheric chemistry.

For spectral calibration the well-known Fraunhofer lines in either the direct sun irradiance or in the Earth Radiance can be used. A manmade option, which to our knowledge does not yet exist, is to implement a solution by using a doping material in the calibration areas.

The concept considered viable to radiometrically calibrate a compact is system is based on a calibration unit that illuminate the spectrometers by-passing the telescope. This solution can easily be achieved by collecting the sun light on an small optical system and transported via an optical fiber to a second slit. The optical system of the calibration unit can be exposed to the Sun light by maneuvering the satellite when the satellite is exiting the eclipse. The calibration unit can be positioned on the satellite to point the cold space during nominal operation. A proper design of the collecting optics and of the relevant baffling will prevent that stray-light will hamper the measurements during nominal operations.

There is a drawback for this solution: any degradation of the telescope and/or the optical fiber will not be detected, as the calibration unit uses a different light path with the respect to the nominal operation. Any degradation of these two units 
will directly impact the absolute radiometric accuracy, as the Sun irradiance measured by the calibration is used to offset the periodical variations of the Sun irradiance. An easy way to circumvent this problem is to maneuver the satellite to point the calibration unit to one of the vicarious calibration sites and compare the radiance measured by the calibration port with the radiance measured by the instrument. The ratio between the two measurements can be compared with the measurements performed on ground. Even if the radiance of the vicarious calibration is know only with $3 \%$ accuracy, it will provide accurate differential measurement of the throughput of the calibration unit with respect to the instrument in nominal operation.

There are also other solutions that could be further investigated, as for example, over-dimensioning the telescope pupil, and use part of it for the calibration, and still use an optical fiber to bring the Sun light during the calibration. The final configuration will be selected at an advanced level of the design. The Authors are confident that, in the assumption that the hosting satellite can provide some level of agility, a configuration with non moving part can easily be implemented.

\subsection{Detector}

The spectral channels ranging from UV to NIR of spectrometers for space applications, such as TROPOMI, Sentinel 4 and 5, are equipped with CCD detectors, and with Mercury Cadmium Telluride (MCT) detectors for the spectral range covering the SWIR part of the spectrum.

The requirements for a CCD detector stemming from a spectrometer working in pushbroom configuration are quite demanding: the readout frequency shall be in the excess of one megahertz to be capable to read the full detector within the time span between two acquisition, the vertical transfer of photo-electron shall also be performed at high speed to reduce the effect of image smearing. Furthermore, the full well of the single pixels shall also be high enough to guarantee the required dynamic range. All these requirements combined together make the detector and its associated electronics very complex and costly.

Recent developments of fast and low noise CMOS detector offer the possibility to simplify the detection chain. CMOS with format of $2 \mathrm{k} \mathrm{x} 2 \mathrm{~K}$ able to run at more than 20 frames per second are readily available on the market. The small full well capacity of a CMOS with respect to $\mathrm{CCD}$ can be easily compensate by reading more frames during a single acquisition. The lower dynamic range can be recovered by using a telescope with a smaller field of view and a more favorable F-number.

For what concerns the detectors of the SWIR channel, recent development of InGaAs technology provide a very appealing alternative to MCTs, as InGaAs can be operated at temperature significantly higher than MCT, reducing the requirements to the cooling system. Unfortunately, the current InGaAs technology is not currently capable of offering detectors with a read-out rate high enough to be used in a push broom instrument, but it is expected that manufacturers of InGaAs detector will soon overcome this limitation. One way to keep the mass of the instrument to an acceptable level, is to use a combination of active and passive cooling system. A solution using last generation mini pulse tube can be accommodated within the power and mass budget suitable for a small satellite.

\subsection{Additive Manufacturing}

Commercial applications have a strong market pull on the additive manufacturing that leads to believe that a constant improvement of accuracy and surface finish will happen in the near future. Therefore it is expected that tight manufacturing tolerance of optomechanical systems will soon be attainable directly with additive manufacturing and/or with some modest post processing. Therefore, in parallel to the optical design and manufacturing, both ESA and TNO are looking into possible applications of additive manufacturing and the advantage it can bring to optical systems. A test run has been performed by TNO to manufacture a sample of the case of one spectral channel (figures 9 and 10). A verification of the accuracies attainable by this first manufacturing run is still in progress, as well as other thermo-elastic analysis to verify the optomechanical coupling of the mirrors with the case. 


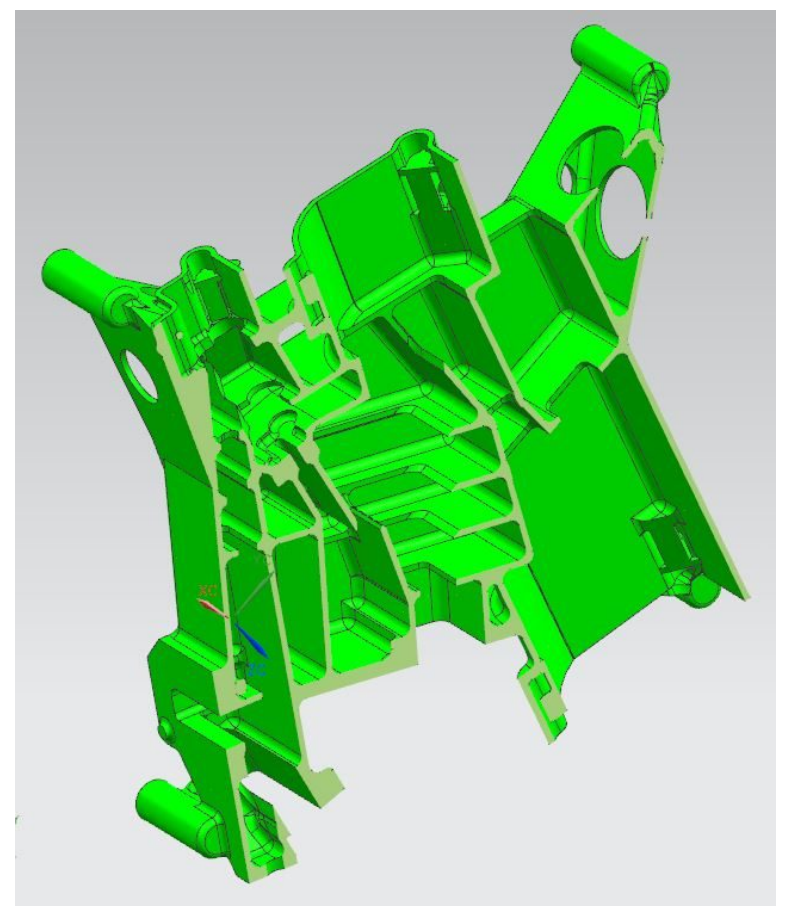

Figure 9 - TROPOLITE Mechanical Design

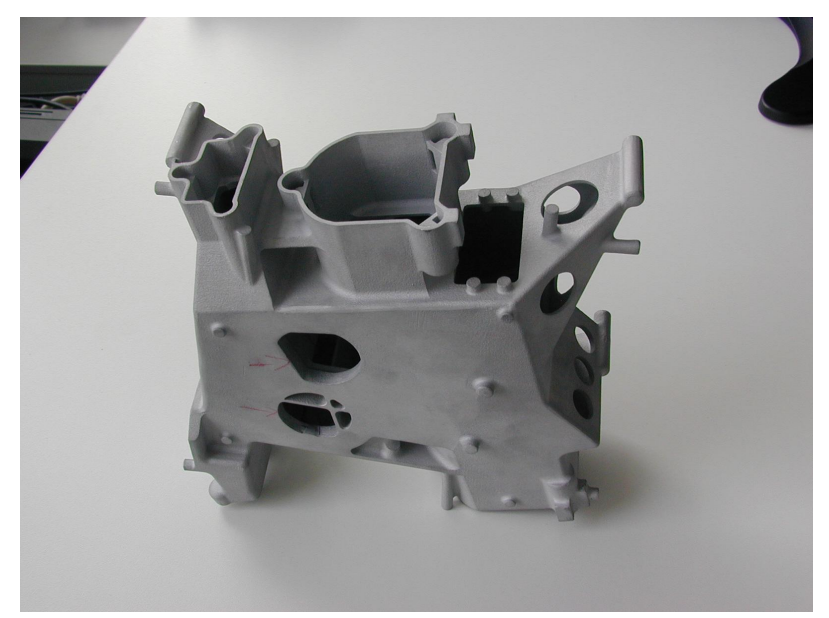

Figure 10 - TROPOLITE case

\section{BASELINE TROPOLITE CONFIGURATION}

A full system trade-off and optimization is still on course. It entails the final selection of the detectors, of the cooling system and the accommodation of the calibration unit. The analysis and the experience gained with TROPOMI shows that waiving the requirement of a field of view of 105 degrees has an impact on performance: a telescope with a smaller field of view gives a better ground sampling distance, a better straylight, and gives a SNR to meet the requirements against which TROPOMI has been designed. 


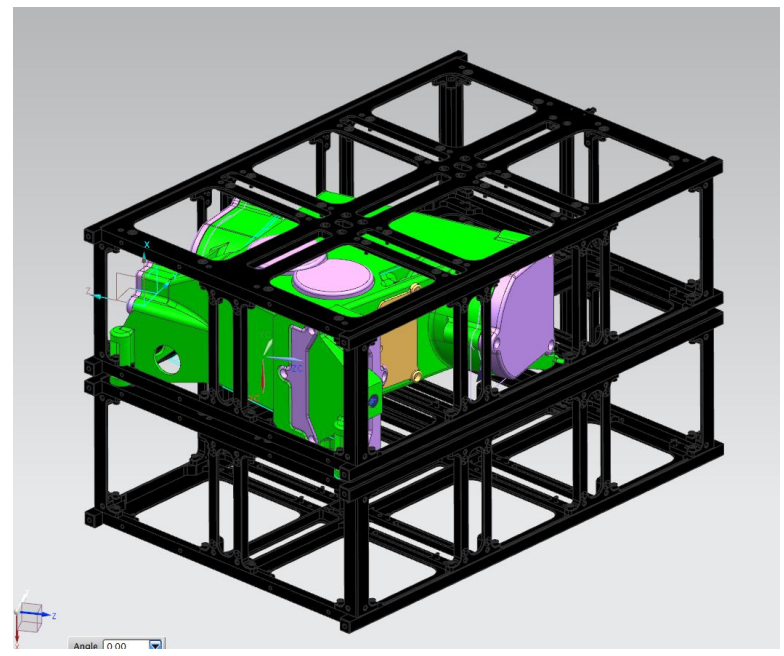

Figure 11 - One TROPOLITE spectral channel inside a twelve units CubeSat

The analysis performed with the TROPOLITE study shows that an optimal solution between complexity and performance has a field of view of between 50 and 70 degrees, giving a swath between 560 and $840 \mathrm{~km}$ from a polar orbit of $600 \mathrm{~km}$ altitude. The full coverage can easily be recovered by launching two or more satellites and at the same obtaining an overall system that is more robust than a single instrument with a large swath.

An accommodation study shows that one single channel could fit within the volume of $10 \times 20 \times 20 \mathrm{~cm}^{3}$ (Figure 11). An instrument with three UV / Visible / NIR spectral channels, namely the same spectral bands of TROPOMI, will therefore be contained within a volume of $20 \times 20 \times 30 \mathrm{~cm}^{3}$, well within the capabilities of a small satellite. The SWIR channel will need to use an immerse grating, as done in the SWIR channel of TROPOMI, to limit the size of the grating. The SWIR channel can use a similar optical lay-out visible channels, with some adaptation to accommodate the use of different diffraction orders. It is expected that a system containing a SWIR channel will exceed the constraint of $20 \times 20$ $\mathrm{x} 40 \mathrm{~cm}^{3}$, and will anyway be addressed at a later stage of the project.

\section{CONCLUSIONS AND OUTLOOK}

The results of this conceptual study show the level of miniaturization and modularity the new technologies can bring and encourages to perform the next step, namely to start manufacturing a demonstration model. The capability to deliver a small and affordable instrument that could be flown on a small satellite opens this type of applications to a significantly wider user community. Instruments for atmospheric chemistry are believed to be next frontier for small satellites: instrument flown so far required large platforms and have been too costly to be within reach of the small satellite community. A number of commercial users are increasingly looking for data on air quality and better knowledge of troposphere chemical processes, provided that they could easily accessible. The work performed so far jointly by ESA, NSO and TNO put TROPOLITE on the leading edge of this type of miniaturized instruments. The path of making measurements of atmospheric chemistry from space simple (and affordable) has been opened. Nevertheless, some challenges still lay ahead: instruments like TROPOLITE produces data in excess of 1TB per orbit, well beyond the current downlink capabilities of small satellites. Recent developments of satellite to ground optical links [4] show that the bottleneck of the data link could be soon resolved. The algorithms and the data processing for the retrieval of the physical parameters from the raw data are quite complex and still subject of research and development. TROPOMI will surely produce the knowledge that will be needed to operate spectrometers for atmospheric chemistry. Once TROPOMI operations will be on course, it will be possible to understand how to streamline the operations to reach a wider user base. As for many Space missions, the Space segment is only a fraction of the overall mission cost. Long term archiving and retrieval of large quantity of data is representing a non-negligible part of the overall mission cost. At the same time, 
the era of publicly sharing large quantity of data is well under course, the booming of social networking being one example. Leveraging on these technologies it will be possible to make access to data to a wide user. To work toward simplicity and usability of these type of date will surely be one of the great challenges for a TROPOLITE systems will have to overcome.

\section{ACKNOWLEDGEMENTS}

The work presented on this paper leverages on many development and analysis performed in the TROPOMI and Sentinel 5P project. The Authors are grateful to the many persons working on the TROPOMI project at Dutch Space, TNO, KNMI and SRON for the open and frank discussions to identify possible pitfall that a 'lighter' version of TROPOMI may have. A special thank goes to Bryan de Goeij, and Gerard Otter for providing valuable technical input and to Bart Snijders and Andy Court for believing in and supporting the idea of developing a 'light version' of TROPOMI.

\section{REFERENCES}

[1] Johan de Vries ; Robert Voors ; A'gnes Mika ; Gerard Otter ; Nick van der Valk ; Ilse Aben ; Ruud Hoogeveen ; Annemieke Gloudemans ; Marcel Dobber ; Pepijn Veefkind and Pieternel Levelt, "TROPOMI, the solar backscatter satellite instrument for air quality and climate, heads towards detailed design" Proc. SPIE 7474, (2009).

[2] André M. Hoogstrate ; Casper van Drunen ; Bart van Venrooy and Rens Henselmans, "Manufacturing of highprecision aspherical and freeform optics" Proc. SPIE 8450, (2012).

[3] Jean-Baptiste Volatier ; Stefan Baümer ; Bob Kruizinga ; Rob Vink, "Case study: TROPOLITE" Proc. SPIE 9131, (2014).

[4] Paul Roßmann, "Potential of Optical Communication Systems for Nanosatellites" Proc. of Small Satellite Systems and Services Symposium, (2014). 